model of human lymphoproliferative disease ${ }^{6}$. Whether BCL-2 overexpression will emerge as a determinant of clinical resistance to rapamycin therapy remains to be seen in ongoing clinical trials of mTOR inhibitors for cancer.

In addition to providing insights into signaling in the mTOR branch of the phosphatidylinositol 3-kinase (PI3K)-Akt pathway, Majumder et al. took steps towards creating a biological readout of RAD001 activity, by iden-

๖ tifying a molecular signature of its action (Fig. 1). They rigorously correlated the biologic drug effects with markers of mTOR bioactivity in studies of nearly 300 Akt-transgenic and wild-type mice. This evaluation included the determination of peak and trough RAD001 levels in tumor tissue and blood, examination in tumor tissue of phosphorylation of downstream mTOR targets (ribosomal protein S6, eukaryotic translation initiation factor $4 G$ ), and detailed analysis of changes in gene expression over time.

Prostates from transgenic mice were enriched for mRNAs regulated by the hypoxia-

윽 inducible factor HIF- $1 \alpha$, and this signature was collectively lost after treatment with RAD001, fully consistent with the known regulation of 글 HIF- $1 \alpha$ by PI3K-Akt signaling in cancer cell lines ${ }^{7}$. The most prominently enriched HIF1- $\alpha$ target genes encoded enzymes of the glycolytic pathway. The same enzymes are responsible for uptake and retention of the positron emission tomography (PET) tracer $\left[{ }^{18} \mathrm{~F}\right] 2$-fluoro-2deoxy-D-glucose (FDG). This observation thus raises the intriguing possibility that FDG-PET scanning may be able to provide noninvasive readouts of mTOR activity in tumors. (FDGPET has been used successfully to monitor the response of gastrointestinal stromal tumors to imatinib, but the mechanism is unknown.)

Successful clinical deployment of targeted cancer therapeutics hinges on the ability to recognize the molecular phenotype of tumors likely to respond to therapy and to monitor target inhibition in tumor tissue during treatment to guide dose selection and interpret clinical results. This paradigm has already met with some success; for instance, frequent sampling of leukemia cells in peripheral blood and bone marrow enabled the investigation of resistance to the BCR-ABL kinase inhibitor imatinib in leukemia ${ }^{8}$. Most solid tumors are much less accessible for repeat biopsies and therefore require the identification of biomarkers that provide noninvasive readouts for pathway activation and drug-target interaction in tumor tissue, preferably through blood tests and imaging modalities.

The urgent need for such biomarkers is illustrated by the recent experience with EGFR kinase inhibitors for lung cancer. Although $10-20 \%$ of patients appeared to respond to therapy in early clinical trials, subsequent trials with over 2,000 patients failed to provide any insights into how to select patients most likely to respond, jeopardizing further clinical development of these compounds ${ }^{9}$. The recent |identification of point mutations in the EGFR kinase domain in tumors from patients responding to EGFR kinase inhibitors (see page 577-578 $)^{10,11}$ now provides a logical tool for patient selection and perhaps monitoring of drug resistance.

Whether this paradigm can be extended to other classes of targeted cancer therapeutics remains to be seen. In the meantime, genetically engineered mice may prove to be an invaluable tool to consolidate genome-wide surveys of gene and protein expression into a set of biomarkers that can be monitored using clinically established methodologies.

1. Van Dyke, T. \& Jacks, T. Cell 108, 135-144 (2002).

2. Majumder, P.K. et al. Nat. Med. 10, 594-601 (2004)

3. Neshat, M.S. et al. Proc. Natl. Acad. Sci. USA 98, 10314-10319 (2001).

4. Podsypanina, K. et al. Proc. Natl. Acad. Sci. USA 98 10320-10325 (2001).

5. Aoki, M., Blazek, E. \& Vogt, P.K. Proc. Natl. Acad. Sci. USA 98, 136-141 (2001).

6. Wendel, H.G. et al. Nature 428, 332-337 (2004).

7. Abraham, R.T. Curr. Top. Microbiol. Immunol. 279, 299-319 (2004)

8. Gorre, M.E. et al. Science 293, 876-880 (2001).

9. Dancey, J.E. \& Freidlin, B. Lancet 362, 62-64 (2003).

10. Paez, J.G. et al. Science published online 29 April 2004 (doi: 10.1126/science.1099314).

11. Lynch, T.J. et al. N. Engl. J. Med. (2004).

12. Manning, B.D. \& Cantley, L.C. Trends Biochem. Sci. 28, 573-576 (2003)

13. Jaeschke, A., Dennis, P.B. \& Thomas, G. Curr. Top. Microbiol. Immunol. 279, 283-298 (2004).

\title{
Targeting tumors: seeing the light
}

Positron emission tomography (PET) enables researchers to peer deep into tissues lit up with a radioactive tracer. In the 1 June Nature Biotechnology, Peter Smith-Jones et al. apply the technique to directly evaluate the effects of an antitumor agent on its target.

As the target, the investigators chose the receptor tyrosine kinase HER2, which can power tumor proliferation and survival when overexpressed, amplified or mutated. As the antitumor agent, the researchers chose 17-allylaminogeldanamycin (17-AAG), a derivative of the antibiotic geldamycin. 17-AAG inhibits Hsp90, a chaperone protein that stabilizes HER2 and other key signaling proteins. 17-AAG causes HER2 degradation and tumor shrinkage in mouse models and has recently entered phase 1 clinical trials.

To visualize HER2 degradation in response to 17-AAG, the researchers first created an antibody fragment that binds to HER2. They then attached this fragment via a molecular link to an isotope of gallium. Shown is a mouse with a tumor (circled) before 17-AAG treatment, shortly after injection with the label. By 24 hours after treatment, the investigators could see HER2 disappear (not shown).

The technique should enable rapid, noninvasive evaluation of dosing and activity for a variety of drugs. The researchers plan to test a labeled antibody fragment against the HER2 family member EGFR, the target of the drug gefitinib (see pages 577-578), which induces EGFR internalization and degradation. To use the technique for visualizing intracellular events, Smith-Jones envisions linking a label to a nuclear hormone or other amenable intracellular regulator.

Charlotte Schubert

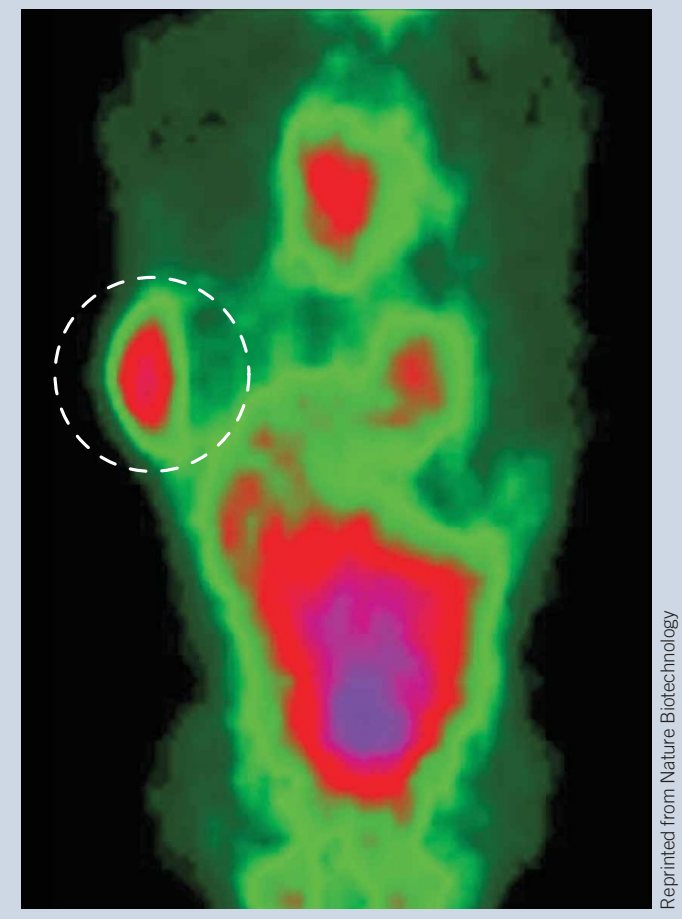

